\title{
Practica Socio-Comunitaria Como Herramienta Para La Articulación Con El Nivel Básico
}

\author{
María José Rosa \\ Valeria Santa \\ Noemí Mónaco \\ Valeria Autrán \\ María Silvana Amín \\ Laura Tamiozzo
}

Facultad de Agronomía y Veterinaria.

Universidad Nacional de Río Cuarto, Argentina

Doi: 10.19044/esj.2017.v13n34p448 URL:http://dx.doi.org/10.19044/esj.2017.v13n34p448

\begin{abstract}
The National University of Río Cuarto, Córdoba, Argentina, called for the incorporation of Socio-Community Practices (PSC) as part of the curriculum in Higher Education. Within the framework of this innovation, the "Socio-Community Practice as a tool for articulation with the initial level at the Jorge Newbery School" was developed within the Agronomic Engineering career. This practice of socio-educational application promotes and makes possible the establishment of social networks and of more inclusive and integrating communities as pedagogical instances of university formation in contexts of solidarity. The objectives were to develop in university students socio-critical attitudes of solidarity, in an interdisciplinary framework with social actors not belonging to their academic environment and at the same time promote in children of the primary school level an ecological conscience, the consumption of healthy foods and organic products from the self-production of vegetables. In different meetings with the children of the Jorge Newbery School, the students of the Agronomic Engineering career developed thematic axes coordinated by a team of interdisciplinary teachers and professionals from the National Institute of Agricultural Technology. This experience generated networks of exchange and enriching instances of the teaching-learning process between students and teachers of differents levels.
\end{abstract}

Keywords: Socio-community practices, university formation, social interaction. 


\section{Resumen}

La Universidad Nacional de Río Cuarto, Córdoba, Argentina, convocó a la incorporación de Prácticas Socio-Comunitarias (PSC) como parte de la currícula en la Educación Superior. En el marco de esta innovación se desarrolló la "Práctica socio-comunitaria como herramienta para la articulación con el nivel inicial en la Escuela Jorge Newbery" dentro de la carrera de Ingeniería Agronómica. Dicha práctica de aplicación socioeducativa promueve y posibilita el establecimiento de redes sociales y de comunidades más inclusivas e integradoras como instancias pedagógicas de formación universitaria en contextos solidarios. Los objetivos fueron desarrollar en los estudiantes universitarios actitudes socio críticas, de solidaridad, en un marco interdisciplinario con actores sociales no pertenecientes a su entorno académico y al mismo tiempo promover en los niños del nivel escolar primario una conciencia ecológica, el consumo de alimentos saludables y orgánicos a partir de la autoproducción de hortalizas. En diferentes encuentros con los niños de la Escuela Jorge Newbery, los estudiantes de la carrera de Ingeniería Agronómica desarrollaron ejes temáticos coordinados por un equipo de docentes interdisciplinario y profesionales del Instituto Nacional de Tecnología Agropecuaria. Esta experiencia generó redes de intercambio e instancias enriquecedoras del proceso enseñanza-aprendizaje entre alumnos y docentes de diferentes niveles.

Palabras clave: Prácticas socio-comunitarias, formación universitaria, interacción social.

\section{INTRODUCCIÓN}

La relación entre la Universidad y la sociedad se concibe como una reciprocidad de comunicación, cooperación y aprendizaje con diversos ámbitos de la comunidad. Estos compendios didácticos se integran como instancias pedagógicas que posibilitan poner en acción conocimientos y competencias adquiridas en los procesos de formación universitaria, en contextos solidarios, pudiendo implementarse tanto en educación formal como no formal. Esto implica un compromiso social de búsqueda de prácticas educativas sociales justas y democráticas (Macchiarola et al., 2012). La carrera de Ingeniería Agronómica tiene entre sus objetivos generales formar profesionales que actúen como agentes de cambio, respondiendo a las demandas y necesidades del contexto social y productivo regional y nacional, movilizadores de las potencialidades de la comunidad y en áreas con niveles de fragilidad económica y social. Esto requiere de la articulación teórico-práctica para la adquisición de capacidades específicas 
bajo diferentes formatos pedagógicos que desarrollan y potencian el "saber hacer y resolver" del futuro profesional.

Entre los beneficios académicos para los estudiantes es de esperar que se logre dominio sobre el contenido de las asignaturas implicadas en la práctica, habilidades de expresión oral y multimedial. Por lo tanto, es necesario generar ámbitos de aprendizaje que promuevan una adecuada percepción de los problemas estructurales en donde se desarrollará la profesión (Edwards, 2004).

En las Prácticas Socio Comunitarias (PSC) los estudiantes universitarios participan en forma voluntaria siendo una instancia de enseñanza -aprendizaje con profunda mirada social (Pozo y Font, 1999). Es importante generar el interés de los estudiantes, ya que el mismo se encuentra condicionado por múltiples variables personales (Paoloni, 2014). Por lo dicho, los docentes universitarios deben intervenir estimulando el interés, generado espacios de participación y de interacción con la sociedad.

Existen diferentes maneras de aprender, las PSC responden al modelo constructivista, en el que el aprendizaje se da como resultado de un proceso de construcción y reconstrucción de significados (Freire, 2004), donde el estudiante es protagonista activo y constructor de su propio aprendizaje, siendo la tarea de los docentes facilitar y orientar en los procesos (Sanjurjo y Vera, 1994). El trabajo interdisciplinario de los docentes es altamente valorable por permitir el intercambio de ideas, materiales, sugerencias y recomendaciones entre colegas. De este modo, se espera que los resultados superen a las capacidades individuales de cada miembro participante (Hernández, 2007).

El cuidado del agroecosistema, desarrollando una cultura agrícola sustentable, manifiesta la necesidad de preservar el medio natural para las actuales y futuras generaciones. Esto requiere formar profesionales con un enfoque socio-humanista (Guzmán Góngora y Cedeño García, 2013), con conciencia ecológica, política, científica, comunicativa, moral y el desarrollo de los valores profesionales. Pensando en las futuras generaciones, en los sectores más vulnerables de nuestra comunidad y en la integración social de los diferentes partícipes que coexisten en ella; dando valor a los saberes propios adquiridos de generación en generación, promoviendo conductas positivas y amigables con el medio ambiente y la economía familiar, se pensó en un equipo interdisciplinario, que integre conocimientos y voluntades, para llevar adelante las PSC. Considerando que los hábitos positivos se adquieren con mayor facilidad en edades tempranas se propuso el presente proyecto para realizar en una escuela de enseñanza general básica.

Los objetivos de la presente PSC son:

Objetivos de la Comunidad educativa de la UNRC 
- Desarrollar actitudes socio críticas, de solidaridad en los estudiantes de la carrera de Ingeniería agronómica en un marco interdisciplinario.

- Fortalecer el sentido crítico de los estudiantes en la interacción activa con actores sociales no pertenecientes a su entorno académico.

- Transferir el conocimiento académico de los estudiantes en la enseñanza de los saberes cotidianos de la comunidad.

- Transmitir respeto por la diversidad biológica entendiendo que no es posible concebir la existencia humana al margen de la naturaleza.

- Proveer los resultados al Colegio "Jorge Newbery" y al Instituto Nacional de Tecnología Agropecuaria (INTA).

Objetivos de Trabajo con la Comunidad educativa "Jorge Newbery":

- Promover el consumo de alimentos saludables y orgánicos a partir de la autoproducción de hortalizas, generando un aporte económico a la familia con conciencia ecológica.

- Adquirir conocimiento acerca de las necesidades en cuanto a la forma de utilización de recursos y herramientas aportadas para la instalación de huertas familiares.

\section{MATERIALES Y MÉTODOS}

Estas prácticas se llevaron a cabo en el transcurso del segundo cuatrimestre del año 2016, con la participación de estudiantes y docentes de la carrera Ingeniería Agronómica que voluntariamente se incorporaron al proyecto de PSC. En la primera etapa los participantes organizaron y prepararon la metodología con que se desarrollaron las diferentes temáticas. Durante encuentros presenciales, que se efectuaron en las instalaciones del colegio Jorge Newbery, los estudiantes universitarios acompañados de los docentes de cada una de las asignaturas que participaron, realizaron una presentación audiovisual y oral interactuando activamente con los niños. En cada encuentro se brindaron talleres con distintos ejes temáticos. En el primero se abordó el tema de la importancia del suelo y del reciclado de la basura como componentes de la huerta orgánica, el rol de los vegetales en la alimentación y de los buenos hábitos alimentarios. Se entregaron bolsitas con compost a cada niño, rotuladas con indicaciones para su utilización. Por último se propusieron actividades de cierre, en las cuales los niños trabajaron en grupos y con la utilización de stickers y láminas donde expresaron lo aprehendido sobre los conceptos vertidos.

En el segundo encuentro se brindó la información necesaria para la siembra de cada especie de importancia hortícola y se le entregaron kits con semillas de hortalizas proporcionadas por el INTA. Con la misma modalidad 
del encuentro anterior se realizó un trabajo práctico con los temas desarrollados ese día.

En el tercer encuentro la temática tratada fue la asociación de especies en la huerta, control biológico de malezas y plagas, además de las propiedades y usos de las plantas aromáticas. Se entregaron bolsitas con estacas y hojas de especies aromáticas, se realizó sorteo de plantines y plantas dado el gran número de niños que participaron (95 niños).

Finalmente, en cada encuentro, se realizaron encuestas a los niños $(n=95)$, directivos $(n=2)$ y a los docentes del establecimiento escolar $(n=4)$, para evaluar las actividades desarrolladas durante la PSC y conocer las inquietudes relacionadas con la siembra de semillas en la huerta. También se realizaron encuestas a los estudiantes participantes $(n=3)$ con el objetivo de evaluar el grado de satisfacción en la participación de las PSC.

\section{RESULTADOS}

Se lograron desarrollar los temas propuestos, observando el interés por los mismos a través de una activa participación e interacción entre los niños y estudiantes que exponían. Los niños lograron trabajar en grupo y realizar las actividades propuestas en forma lúdica. A partir del procesamiento de las encuestas realizadas a los participantes se logró evaluar todo lo actuado.

Las encuestas a los estudiantes de la Universidad que fueron los encargados de llevar adelante el encuentro indican que se le explicaron correctamente los objetivos y alcance de la PSC por parte de los docentes; consideraron adecuados los contenidos seleccionados para las clases y que tanto las herramientas como los temas abordados fueron relevantes, ya que implican un acercamiento a cuestiones fácilmente aplicables a la vida cotidiana de los receptores del mensaje. Destacaron la importancia de entender diversas realidades y devolver a la comunidad la oportunidad que tuvieron de acceder a una educación superior gratuita y de calidad. Conocer otras realidades fuera del ámbito universitario implica una apertura mental hacia otras posibilidades de labor, como así también una gran satisfacción al saber que se puede aportar algo mínimo pero de gran valor para el bienestar de muchas familias. Algunas sugerencias que aportaron para mejorar esta actividad fueron: realizar la charla con grupos más reducidos, enfocar y profundizar en aspectos directos acerca de cómo llevar adelante una huerta familiar, analizar la posibilidad de realizar una huerta en la institución, favorecer la interacción y el intercambio de opiniones con los receptores e involucrar no solo a los niños, sino también a aquellos padres o adultos interesados en la temática.

Del procesamiento de las encuestas realizadas a los niños de la escuela Jorge Newbery, surge como resultado que el total de los alumnos 
manifestó consumir frutas y verduras, que un $50 \%$ de las familias de los alumnos no realiza separación de los residuos; mostrando este resultado la necesidad de transferir conocimientos en estas temáticas. El 93\% de los niños consideró que las charlas desarrolladas en los talleres les sirvieron para conocer la importancia de comer sano, reciclar y realizar huertas familiares y un $88 \%$ de ellos manifestaron interés en recibir más información sobre las diferentes temáticas.

Las autoridades del establecimiento expresaron que fue muy interesante y adecuada la temática y el lenguaje utilizado, cubriendo todas las expectativas. La PSC se desarrolló en forma didáctica, lúdica y amena. Sugirieron, al igual que los estudiantes, trabajar con grupos reducidos de niños, realizar la huerta en el establecimiento y que el proyecto se prolongue en el tiempo.

\section{CONCLUSIÓN}

Es importante implementar en la educación superior procesos que motiven y den importancia a las prácticas socio-comunitarias como estrategia para resaltar valores profesionales, promover una manifiesta responsabilidad social y desarrollar el pensamiento creativo, activo $\mathrm{y}$ solidario. El desarrollo de la actividad de PSC permitió establecer vínculos con directivos y docentes de la escuela Jorge Newbery como eje de futuras actividades de articulación, logrando difundir y generar la inquietud en estudiantes de nivel básico acerca de los beneficios de una alimentación saludable, la autoproducción de hortalizas, la organización de la huerta, la preparación del compost y el uso y propiedades de las plantas aromáticas incorporadas al sistema hortícola. Se logró que los estudiantes universitarios adquieran destrezas para trabajar productiva y solidariamente en pos de la sociedad, identificando las necesidades de la comunidad. Dentro de la universidad se consolidó un equipo de trabajo interdisciplinario entre los docentes participantes y fuera de ella se logró trabajar en cooperación con otras instituciones como el INTA y la escuela Jorge Newbery.

\section{References:}

1. Edwards, M., Vilches, A., Gil, D., \& Praia, J. 2004. La atención a la situación del mundo en la educación científica. Enseñanza de las Ciencias, 22(1), 047-64.

2. Freire, P. 2004. Pedagogía de la autonomía: Saberes necesarios para la práctica educativa. Ed. Siglo vientiuno. ISBN 978-987-629-039-5.

3. Guzmán Góngora, C. D. L. Á., \& Cedeño García, B. T. 2013. La formación sociohumanista del Ingeniero Agrónomo. Un modelo pedagógico. Revista Didasc@ lia: Didáctica y Educación, 4(3). 
4. Hernández, A. L. 2007. 14 ideas clave. El trabajo en equipo del profesorado(Vol. 2). Graó.

5. Macchiarola, V.; C. Martini y A. Montebelli. 2012. Prácticas socio comunitarias en el trayecto formativo del profesional de Educación Especial En: Revista RUEDES, Año 2- No 3- 2012, ISSN: 18535668, p. 61-77.

6. Paoloni, P. V. 2014. Emociones en contextos académicos. Perspectivas teóricas e implicaciones para la práctica educativa en la universidad. Electronic Journal of Research in Educational Psychology, 12(3), 567-596.

7. Pozo, J. I., y Font, C. M. 1999. El aprendizaje estratégico: enseñar a aprender desde el currículo. Santillana.

8. Sanjurjo, L y Vera, M.T. 1994. Aprendizaje significativo y enseñanza en los niveles medio y superior. Rosario: Homo Sapiens. 